\title{
Nosocomial Infections after Peripheral Arterial Bypass Surgery
}

\author{
Arianne Ploeg $\cdot$ Christopher Lange $\cdot$ Jan-Willem Lardenoye $\cdot$ \\ Paul Breslau
}

Published online: 6 June 2007

(C) Société Internationale de Chirurgie 2007

\begin{abstract}
Background Hospital-acquired infections account for a substantial increase in morbidity and mortality. This prospective, single-center observational study was conducted to assess the incidence and analyze the risk factors of nosocomial infection after peripheral arterial bypass surgery.

Methods The incidence of nosocomial infections was registered in all patients undergoing peripheral arterial bypass surgery from January 1996 until December 2004, and risk factors for the development of a nosocomial infection were analyzed.

Results A total of 67 infections were diagnosed in association with 607 procedures, yielding an infection ratio of $10.0 \%$. Surgical site infection was the most common (55.2\%), followed by urinary tract infection (16.4\%), pneumonia (14.9\%) and bacteremia (10.4\%). Staphylococcus aureus was the most commonly found isolate in surgical site infections (48.6\%) and in bacteremia (42.9\%). Age, the use of corticosteroids $(p=0.02)$, and critical ischemia with tissue loss $(p=0.009)$ could be identified as risk factors for the development of a nosocomial infection. Blood transfusion was a postoperative risk factor for nosocomial infection $(p<.0001)$. Nosocomial infection was associated with a prolonged hospital stay $(p<.0001)$.

Conclusions This study provides a detailed description of the incidence and risk factors regarding nosocomial infection. More detailed studies are necessary to develop
\end{abstract}

A. Ploeg $(\bowtie) \cdot$ C. Lange $\cdot$ J.-W. Lardenoye

P. Breslau

Department of Vascular Surgery, Haga Hospital, Sportlaan 600,

The Hague 2566 MJ, The Netherlands

e-mail: arianneploeg@hotmail.com strategies to diminish the occurrence of nosocomial infection.

Hospital-acquired infections pose a continuous threat to the health of hospitalized patients and account for a substantial increase in morbidity and mortality. In addition, they impose an economic burden on the hospital sector through prolonged hospitalization and increased health care costs [1]. Surveillance of nosocomial infections is regarded as an essential part of infection control [2-4]. Various studies have previously reported on nosocomial infections in a hospital setting or after general surgery [4-6]. The most frequently occurring nosocomial infections in surgical patients are surgical site infections, urinary tract infections, pneumonia, and sepsis [5]. The most common infectious complication following peripheral arterial reconstructive surgery, with a reported incidence ranging from $5.3 \%$ to $25 \%[7,8]$, is surgical site infection, which has therefore also received the greatest amount of attention [9]. Yet as the patient population ages [10] and becomes more complex, the incidence and range of nosocomial infections may also have become significantly broader [11]. As previous detailed reports in this area appear to be lacking, the present study was conducted with a view to investigating the incidence and analyzing the risk factors of nosocomial infection after peripheral arterial bypass surgery. These observational data may lead to the development of strategies to control and prevent nosocomial infections.

In this connection, it should be noted that several published studies with a view to improving the quality of care have identified risk factors for the development of a nosocomial infection after general or specific types of surgery. Reported risk factors for nosocomial infection after lung surgery included chronic obstructive pulmonary disease (COPD), duration of surgery, and admission to the intensive 
care unit (ICU) [12]. Risk factors after cardiovascular operations included age above 65 , female sex, mode of surgical intervention, general anesthesia, and blood transfusion [13]. After peripheral arterial bypass surgery, the influence of different risk factors in individual patients remains unclear. If risk factors of nosocomial infections in this specific group of patients could be identified, they may be useful in preventing the occurrence of nosocomial infection and thus in improving the quality of care.

\section{Methods}

This study is a prospective, single-center observational study. All consecutive patients undergoing peripheral arterial bypass surgery were included from January 1996 until December 2004. Patient characteristics registered included age, sex, presence of diabetes, cardiovascular disease, end stage renal disease, COPD, use of steroids at admission to the hospital, current smoking, body mass index, and American Society of Anesthesiologists (ASA) classification.

Perioperative and postoperative data included type and indication of surgery performed, type of bypass used, total length of hospital admission, whether the surgery was performed on an emergency basis, duration of surgical procedure, blood transfusion, blood loss, the use of antibiotics perioperative and postoperative and admission to the ICU.

As antibiotic prophylaxis, $1 \mathrm{~g}$ of cefazolin was administered to all patients $30 \mathrm{~min}$ before the start of surgery. All patients underwent postoperative duplex investigation of the bypass in the recovery room in order to determine the patency of the graft. In this study, graft patency was determined by the initial duplex performed in the recovery room or at discharge.

Patients with nosocomial infections within 30 days from index operation were identified from our prospectively kept complication registration system [7]. Infections were considered nosocomial if they were not documented or suspected at the time of admission. Patients with an infection clearly associated with prior surgery who were discharged home but readmitted for treatment of the infection in question were included as nosocomial infection [11]. Follow-up of all patients took place at our outpatient clinic during a 30-day follow-up period after index operation. Registration of nosocomial infections took place twice a day, during morning and evening ward meetings.

All infections were diagnosed according to the definitions of nosocomial infections used by the Centers for Disease Control and Prevention. Pneumonia was defined as a persistent or progressive infiltrate or consolidation in two or more serial chest radiographs, combined with fever or a new onset of purulent sputum or a change in character of sputum and new onset of cough or worsening of cough. Urinary tract infection was defined as a positive culture from urine combined with clinical signs, such as dysuria or fever. Sepsis was defined as a recognized pathogen cultured from one or more blood cultures, combined with symptoms of systemic response to infection, such as fever and hemodynamic changes.

Surgical wound infection was defined when one of the following occurred: purulent drainage from the superficial incision or organisms isolated from an aseptically obtained culture of the superficial incision [14, 15]. Wound infections were then classified according to Szilagyi: grade I, dermis intact (cellulitis, seroma, serous leak); grade II, dermis not intact (necrosis, dehiscence, and abscess); and grade III, graft exposed [16]. All obtained cultures were identified from the hospital data-collection system. Furthermore, all antibiotics administered to each patient were noted.

Statistical analysis was performed using the chi-square test for discrete variables; Fisher's exact test for small numbers, and t-test for continues variables. All differences were considered significant at $p<0.05$.

\section{Results}

Procedures

A total of 607 procedures were performed from January 1996 until December 2004. The mean age of the patients was 70.3 years (range: 20-96 years); 347 were men $(57.2 \%)$. Of the bypass procedures performed, 290 (47.8\%) were suprageniculate, 137 (22.5\%) infrageniculate, 53 $(8.7 \%)$ femorofemoral, and $99(16.3 \%)$ femorocrural. The remaining 28 procedures were more complicated, and consisted, for example, of a combination of a central reconstruction combined with a suprageniculate bypass procedure. Polytetrafluoroethylene (PTFE) grafts were placed in $420(69.2 \%)$ cases, and vein grafts were use in $162(26.7 \%)$ cases; other grafts, including a human umbilical cord graft, were used in the other $24(39.5 \%)$ procedures. Severe claudication was the indication for surgery in 239 (39.4\%) patients, ischemic rest pain in 231 (38.1\%) patients, and ischemic tissue loss in 129 (21.3\%) patients. In 8 patients, the indications were varied, and included, for example, a popliteal aneurysm. Sixty-seven procedures were emergent procedures. Mean hospital stay was 17 days (range: 2-251 days). The overall 30-day mortality was $2.8 \%$. 
Nosocomial infection

Nosocomial infection occurred in association with 61 procedures. A total of 67 infections were diagnosed in 607 procedures, yielding an infection ratio of $10.0 \%$. Most nosocomial infections occurred in relation to a surgical site infection (55.2\%), with an infection ratio of 37/607 (6.1\%). Thirteen surgical site infections $(35.1 \%)$ were diagnosed after the patients were discharged from the hospital; all of them required that the patient be readmitted. Polytetrafluoroethylene grafts were used in 26 patients who developed a surgical site infection (70.3\%), vein grafts $(27.0 \%)$ in two, and an allograft $(2.7 \%)$ in one. Of all surgical site infections, three were considered Szilagyi class III. In one patient, the bypass was removed and an iliofemoral bypass procedure was performed, and in another, a gracilis muscle graft was used to cover the bypass. Limb salvage was achieved in both patients. One patient required an aboveknee amputation. In eleven cases, the surgical site infection occurred after discharge and patients were readmitted to the hospital. In this group, all infections were considered Szilagyi I.

Urinary tract infection was the second most common nosocomial infection seen, occurring in $16.4 \%$ of all cases, followed by pneumonia $(14.9 \%)$ and bacteremia $(10.4 \%)$. In one case, the nosocomial infection consisted of cholecystitis and in another, phlebitis of the hand.

A total of 16 different species of pathogens were isolated. Staphylococcus aureus was the most commonly found isolate in surgical site infections (48.6\%) and in bacteremia $(42.9 \%)$. Furthermore, Staph. aureus was isolated in $20 \%$ of all nosocomial pneumonia and urinary tract infections. In six patients, no culture was obtained (4 surgical site infections, 1 case of pneumonia, and 1 urinary tract infection; Table 1).

As antibiotic prophylaxis, $1 \mathrm{~g}$ of cefazolin was administered to all patients $30 \mathrm{~min}$ before the start of surgery. In the group in which no infections occurred, 69 of the patients had received antibiotics after the index operation, but the criteria for a surgical site infection were not met; for example, no pathogen could be identified from the culture obtained. Of this group without infections, 58 patients had received a PTFE graft; 7 patients, a vein graft; and in 4 patients, other graft types were placed.

\section{Risk factors}

Analysis of a number of preoperative variables (Table 2) revealed that age, the use of corticosteroids $(p=0.02)$, and critical ischemia with tissue loss $(p=0.009)$ were risk factors for the development of a nosocomial infection. No intraoperative risk factors (Table 3) could be identified. Analysis of postoperative variables showed that blood transfusion was a risk factor for nosocomial infection $(p<$ .0001). Nosocomial infection was associated with a prolonged hospital stay $(p<.0001)$.

\section{Discussion}

This study proposed to examine the incidence and type of nosocomial infections documented after peripheral arterial bypass surgery. We have no knowledge of any previous studies in this specific type of patient population. The incidence of nosocomial infection was $10.0 \%$ in patients undergoing peripheral arterial bypass surgery. This is well within the range of $1.4 \%$ to $17.3 \%$ [5] previously reported for general surgery. The distribution of nosocomial infections was, however, found to be different from that in general surgery [4]. After general surgery, urinary tract infections, pneumonia, surgical site infection, and bacteremia are the leading types of infection reported, in that order. Previous studies in which all surgical wounds were observed showed an overall incidence of surgical site infection of $4.7 \%$ [17, 18]. In our study, surgical site infection, with an incidence of $6.1 \%$, was the most frequent. This is somewhat lower than the 7\% $-44 \%[19,20]$ reported in the literature after infrainguinal bypass surgery. Of all surgical site infections, $35.1 \%$ were diagnosed after the patients were discharged from the hospital, a finding that emphasizes the need to develop dependable methods, which should include a reliable postoperative 30-day follow-up, for identifying accurate numbers of surgical site infection [12]. The lower rate of urinary tract infections in our study may be explained by the systematic use of antibiotic prophylaxis on withdrawal of the catheter and a retention protocol [21].

A wide variety of infecting organisms was found, but the importance of Staph. aureus as a pathogen in nosocomial infections is emphasized here. It was the most common isolate in surgical site infection (48.6\%) and in bacteremia (42.9\%). No methicillin-resistant Staph. aureus (MRSA) was isolated, probably because of the very strict Dutch policy to eradicate MRSA, a policy that is far more stringent than those employed in other countries. It has proven to be successful epidemiologically with an incidence of MRSA in the Netherlands <0.5\% [22] Most nosocomial infections were cultured, but in 8 infections, no culture was obtained. Vazquez et al. reported a diagnosis in $67.2 \%$ of all nosocomial infections treated with antibiotics [5]. Another report showed that as many as $68 \%$ of all wound infections was treated without culture [10].

The mean age of the patients in our study was relatively-although not unexpectedly-high, in view of the type of population concerned: an aging population of patients with atherosclerosis undergoing peripheral arterial 
Table 1 Pathogens

\begin{tabular}{|c|c|c|c|c|c|}
\hline Pathogen & $\begin{array}{l}\text { Bacteremia } \\
(n=7)\end{array}$ & $\begin{array}{l}\text { Pneumonia } \\
(n=10)\end{array}$ & $\begin{array}{l}\text { Surgical site } \\
\text { infection }(n=37)\end{array}$ & $\begin{array}{l}\text { Urinary tract } \\
\text { infection }(n=11)\end{array}$ & Other \\
\hline Staph. aureus & 3 & 2 & 18 & 2 & \\
\hline Staph. epidermidis & & & 2 & & \\
\hline Pseudomonas aeruginosa & 1 & & 3 & 1 & \\
\hline Mixed flora & & & 1 & 2 & \\
\hline Proteus mirabilis & 1 & & 2 & & \\
\hline Streptococcus haemolyticus & & & 5 & 1 & \\
\hline Klebsiella spp. & & & & 1 & \\
\hline Strep. pneumoniae & & 2 & & & \\
\hline Escherichia coli & & 1 & 1 & 2 & \\
\hline Enterococcus spp. & & & 1 & 2 & \\
\hline Enterobacter spp. & & 1 & 2 & & \\
\hline Serratia spp. & 1 & & & & \\
\hline Branhamella spp. & & 2 & & & \\
\hline Citrobacter spp. & & 1 & & & \\
\hline No culture obtained & & 1 & 4 & 1 & 2 \\
\hline No pathogen identified & 1 & 1 & 4 & & \\
\hline
\end{tabular}

Table 2 Patient demographics and preoperative factors

\begin{tabular}{|c|c|c|c|c|c|}
\hline & $\begin{array}{l}\text { Infection } \\
\text { group }(n=61)\end{array}$ & & $\begin{array}{l}\text { Non-infection } \\
\text { group }(n=546)\end{array}$ & & $p$ Value \\
\hline Mean age (years) & 70.0 & & 73.3 & & 0.044 \\
\hline Gender ( $\%$ male $)$ & 29 & 47.5 & 318 & 58.2 & 0.11 \\
\hline Mean BMI & 25.1 & & 24.8 & & 0.62 \\
\hline Cardiac disease $(\%)$ & 23 & 37.7 & 195 & 35.7 & 0.76 \\
\hline COPD $(\%)$ & 14 & 22.6 & 96 & 17.6 & 0.30 \\
\hline Diabetes mellitus (\%) & 21 & 34.4 & 134 & 24.5 & 0.093 \\
\hline CVA $(\%)$ & 4 & 6.6 & 61 & 11.2 & 0.27 \\
\hline ESRD $(\%)$ & 3 & 4.9 & 20 & 3.7 & 0.62 \\
\hline Medical steroid use & 9 & 14.8 & 35 & 6.4 & 0.020 \\
\hline Current smokers (\%) & 29 & 47.5 & 335 & 61.4 & 0.14 \\
\hline ASA I $(\%)$ & 3 & 4.9 & 25 & 4.6 & 0.90 \\
\hline ASA II (\%) & 31 & 50.8 & 338 & 61.9 & 0.09 \\
\hline ASA III (\%) & 23 & 37.7 & 171 & 31.3 & 0.31 \\
\hline ASA IV (\%) & 3 & 4.9 & 12 & 2.2 & 0.19 \\
\hline Other indication (\%) & 1 & 1.6 & 7 & 1.3 & 0.81 \\
\hline Severe claudication $(\%)$ & 19 & 31.1 & 220 & 40.3 & 0.166 \\
\hline Ischemic rest pain (\%) & 20 & 32.8 & 210 & 38.5 & 0.386 \\
\hline Ischemic tissue loss (\%) & 21 & 34.4 & 109 & 20.0 & 0.009 \\
\hline
\end{tabular}

$B M I$ body mass index; COPD chronic obstructive pulmonary disease; ESRD end stage renal disease; CVA cerebrovascular accident; $A S A$ American Society of Anesthesiologists

bypass surgery. Older age was found to be statistically significantly associated with a higher prevalence of nosocomial infections, a finding that has been previously demonstrated by other studies [13]. Of all variables studied, the preoperative use of corticosteroids was a risk factor for the development of a nosocomial infection. The incidence of nosocomial infection was not significantly higher in obese, diabetic, or smoking patients, probably because of the high prevalence in both groups. Another feature, ASA physical status grouping, has been shown to be predictive 
Table 3 Perioperative and postoperative factors

\begin{tabular}{|c|c|c|c|c|c|}
\hline & $\begin{array}{l}\text { Infection group } \\
(n=61)\end{array}$ & & $\begin{array}{l}\text { Non-infection } \\
\text { group }(n=546)\end{array}$ & & $p$ Value \\
\hline Suprageniculate (\%) & 26 & 42.6 & 266 & 48.7 & 0.38 \\
\hline Infrageniculate $(\%)$ & 16 & 26.2 & 121 & 22.1 & 0.47 \\
\hline Femorofemoral (\%) & 2 & 3.3 & 51 & 9.3 & 0.027 \\
\hline Femorocrural (\%) & 16 & 26.2 & 83 & 15.2 & 0.11 \\
\hline Other $(\%)$ & 1 & 1.6 & 25 & 4.6 & 0.28 \\
\hline PTFE & 40 & 65.6 & 380 & 69.6 & 0.52 \\
\hline Vein & 17 & 27.9 & 145 & 26.6 & 0.83 \\
\hline Other $(\%)$ & 3 & 4.9 & 21 & 3.8 & 0.73 \\
\hline Acute operation (\%) & 2 & 3.3 & 14 & 2.6 & 0.99 \\
\hline Re-incision (\%) & 16 & 26.2 & 181 & 33.2 & 0.27 \\
\hline Duration of operation (min) & 118.9 & & 114.2 & & 0.22 \\
\hline Mean blood loss (ml) & 82 & & 94 & & 0.69 \\
\hline Hospital stay (days) & 28 & & 18 & & $<.0001$ \\
\hline Patency of graft (\%) & 53 & 86.9 & 504 & 92.3 & 0.14 \\
\hline Blood transfusion & 17 & 27.9 & 44 & 8.1 & $<.0001$ \\
\hline Admittance ICU & 8 & 13.1 & 38 & 7.0 & 0.084 \\
\hline 30-day mortality & 0 & 0 & 17 & 3.1 & 0.24 \\
\hline
\end{tabular}

PTFE polytetrafluorethylene; $I C U$ intensive care unit

of postoperative wound infections in surgical patients, although in this study a higher ASA class could not be associated with a higher prevalence of infection [12].

Patients suffering from ischemic tissue loss also showed a higher prevalence of nosocomial infection. Not only does tissue loss offer a passage of an entry for pathogens, patients with tissue loss, in general, tend to be in poorer physical condition preoperatively.

Of all operations performed, a femorofemoral bypass graft was found to be associated with a relatively low incidence of nosocomial infections. Chang reported a similar result, with $0.3 \%$ incidence of surgical site infection after the femorofemoral bypass procedure [20]. Although a relatively high number of PTFE grafts were placed in our study, there was not an associated increase in graft infections, notwithstanding all suggestions made in earlier studies to the contrary. Graft infections do not necessarily appear in the immediate postoperative course. Lorentzen et al. reported an incidence of $53 \%$ within 30 days of all graft infections observed [2]. Similarly, we were unable to demonstrate an increase in superficial surgical site infections in association with the use of an autogenous vein graft [20].

Among the 3 Szilagyi class III infections, limb salvage could not be achieved in one case. Duration of the operation was not identified in this study as a risk factor, although it is widely accepted that operations longer than $2 \mathrm{~h}$ are associated with a higher prevalence of surgical site infection. Likewise, the amount of blood loss could not be identified as a risk factor, but administration of any blood transfusion was identified as risk factor for nosocomial infection, as previously described by Rebollo et al. [13]. Reoperation was not a risk factor for the development of a nosocomial infection.

Nosocomial infection was not associated with an increase in 30-day mortality, probably because of the low incidence of mortality after peripheral arterial bypass surgery. Prolonged admission time to the hospital was associated with a statistically significant rise in nosocomial infections and thus reflects the increase in economic costs and morbidity for the patient.

In conclusion, this study provides a detailed description of the incidence and risk factors regarding nosocomial infection. Older age, the use of corticosteroids, and the presence of tissue loss were intrinsic patient factors found to be associated with nosocomial infection. The administration of a blood transfusion could be identified as an extrinsic risk factor.

It may be conjectured that older patients with ischemic tissue loss who receive corticosteroids, should be carefully monitored during their hospital stay. Also, postoperative prophylactic antibiotics should be given serious consideration. More detailed studies are necessary to identify risk factors in the complex management of this group of patients and to develop strategies to diminish the occurrence of nosocomial infection. 


\section{References}

1. Plowman R, Graves N, Griffin MA, et al. (2001) The rate and cost of hospital-acquired infections occurring in patients admitted to selected specialties of a district general hospital in England and the national burden imposed. J Hosp Infect 47:198-209

2. Lorentzen JE, Nielsen OM, Arendrup H, et al. (1985) Vascular graft infection: an analysis of sixty-two graft infections in 2411 consecutively implanted synthetic vascular grafts. Surgery 98:8186

3. Haley RW, Schaberg DR, McClish DK, et al. (1980) The accuracy of retrospective chart review in measuring nosocomial infection rates. Results of validation studies in pilot hospitals. Am J Epidemiol 111:516-533

4. Eriksen HM, Iversen BG, Aavitsland P. (2005) Prevalence of nosocomial infections in hospitals in Norway, 2002 and 2003. J Hosp Infect 60:40-45

5. Vazquez-Aragon P, Lizan-Garcia M, Cascales-Sanchez P, et al. (2003) Nosocomial infection and related risk factors in a general surgery service: a prospective study. J Infect 46:17-22

6. Horan TC, Culver DH, Gaynes RP, et al. (1993) Nosocomial infections in surgical patients in the United States, January 1986June 1992. National Nosocomial Infections Surveillance (NNIS) System. Infect Control Hosp Epidemiol 14:73-80

7. Schepers A, Klinkert P, Vrancken Peeters MP, et al. (2003) Complication registration in patients after peripheral arterial bypass surgery. Ann Vasc Surg 17:198-202

8. Kent KC, Bartek S, Kuntz KM, et al. (1996) Prospective study of wound complications in continuous infrainguinal incisions after lower limb arterial reconstruction: incidence, risk factors, and cost. Surgery 119:378-383

9. Goldstone J, Moore WS. (1974) Infection in vascular prostheses. Clinical manifestations and surgical management. Am J Surg 128:225-233

10. Rothwell PM, Coull AJ, Silver LE, et al. (2005) Population-based study of event-rate, incidence, case fatality, and mortality for all acute vascular events in all arterial territories (Oxford Vascular Study). Lancet 366(9499):1773-1783
11. Sawyer RG, Raymond DP, Pelletier SJ, et al. (2001) Implications of 2,457 consecutive surgical infections entering year 2000. Ann Surg 233:867-874

12. Nan DN, Fernandez-Ayala M, Farinas-Alvarez C, et al. (2005) Nosocomial infection after lung surgery: incidence and risk factors. Chest 128:2647-2652

13. Rebollo MH, Bernal JM, Llorca J, et al. (1996) Nosocomial infections in patients having cardiovascular operations: a multivariate analysis of risk factors. J Thorac Cardiovasc Surg 112:908-913

14. Vrancken Peeters MP, Vrancken Peeters MJ, Corion LU, et al. (2005) Quality control of colorectal surgery with an extensive complication registration system. Dig Surg 22:168-173

15. Horan TC, Gaynes RP. (2004) Surveillance of Nosocomial Infections. Hospital Epidemiology and Infection Control, 3rd Edition, Philadelphia, Lippincott Williams \& Wilkins, 1659-1702

16. Szilagyi DE, Smith RF, Elliott JP, et al. (1972) Infection in arterial reconstruction with synthetic grafts. Ann Surg 176:321333

17. Cruse PJ, Foord R. (1980) The epidemiology of wound infection. A 10-year prospective study of 62,939 wounds. Surg Clin North Am 60:27-40

18. Cruse PJ, Foord R. (1973) A five-year prospective study of 23,649 surgical wounds. Arch Surg 107:206-210

19. Lee ES, Santilli SM, Olson MM, et al. (2000) Wound infection after infrainguinal bypass operations: multivariate analysis of putative risk factors. Surg Infect (Larchmt) 1:257-63

20. Chang JK, Calligaro KD, Ryan S, et al. (2003) Risk factors associated with infection of lower extremity revascularization: analysis of 365 procedures performed at a teaching hospital. Ann Vasc Surg 17:91-96

21. Burger DH, Kappetein AP, Boutkan H, et al. (1997) Prevention of urinary retention after general surgery: a controlled trial of carbachol/diazepam versus alfusozine. J Am Coll Surg 185:234-236

22. Vriens M, Blok H, Fluit A, et al. (2002) Costs associated with a strict policy to eradicate methicillin-resistant Staphylococcus aureus in a Dutch University Medical Center: a 10-year survey. Eur J Clin Microbiol Infect Dis 21:782-786 